\title{
Research and Application of MES and ERP System Integration Methods in Steel Industry
}

\author{
Xuejiao Tian ${ }^{1+}$, Zixuan $\mathrm{Wu}^{1,2}$, Jianjian Wang ${ }^{1}$ and $\mathrm{Wu}$ Yang ${ }^{1}$ \\ ${ }^{1}$ Donlinks School of Economics and Management, University of Science and Technology Beijing, Beijing \\ 100083, China \\ ${ }^{2}$ Ministry of Education Engineering Research Center of MES Technology for Iron\&Steel Production, \\ Beijing 100083, China
}

\begin{abstract}
With the development of manufacturing environment, the connotation and model of the information system construction of manufacturing enterprises are constantly changing. To achieve the information strategic target of control \& management integration and production-marketing integration, the development and integration of the information system of the high-level planning system and the manufacturing execution level have become the top priority of enterprise information construction. Based on the information construction of a large steel mills in northwest China, an information integration environment that takes into account the characteristics of enterprise management and production processes is proposed using modular design. The final implementation results prove the reliability and stability of the system, and the accuracy and completeness of the transaction data.
\end{abstract}

Keywords: management information systems, manufacturing execution system, enterprise resource planning, system integration

\section{Introduction}

The manufacturing execution system (MES) was first formally proposed by the Advanced Manufacturing Research (AMR) in the 1990s. It is a system of two-way data interaction between the enterprise resource planning (ERP) and the process control system (PCS) of the workshop level [1 2]. In recent years, as an important part of the information management of manufacturing enterprises, MES has received more and more attention. Nan [3] indicated that, an excellent MES requires not only its own reconfigurability and adaptability, but also its independence and compatibility with other systems. The research of MES must be incorporated into the research of enterprise information construction [4]. In addition, with the product functions become more and more abundant, and production automation gradually improved, a lot of information needs to be processed by MES [5]. It is difficult to satisfy the enterprise's actual demand. Therefore, it's essential to extend the MES and implement the interface of MES and ERP (MES-ERP).

The main function of MES-ERP interface is to achieve information interaction between heterogeneous systems, closely link business logic between heterogeneous systems, and support a complete closed loop business process [6]. The key points are: (1) The integration of MES and ERP is, in essence, the problem of industrial control and management control; (2) ERP production emphasizes overall macro-control, while MES is in the middle of the manufacturing implementation level and has ability to operate independently, so the balance between system role sharing and function execution is very important; (3) On account of the rapid iteration speed of information construction, the system design should be flexible and developable from

\footnotetext{
+ Corresponding author. Tel.: +86 18811345560 ; fax: +861062334598.

E-mail address: tianxuejiao1990@126.com.
} 
the cost perspective; (4) How to guarantee the timeliness and accuracy of data interaction between two systems.

The mainly contributions in this paper are as follows: MES uses two-layer architecture to emphasize independence and compatibility, the MES-ERP interface integration adopts different interface architecture design methods according to different modules, such as background service, data message, and program reconfiguration, etc., which can provide reference for MES system environment design. Furthermore, on the basis of the above analysis and previous studies, we take the information system integration of a large steel plant in northwest China as the background, further optimize the management mode by means of information technology, breakthrough the information gap between MES and ERP, complete the information interaction between different hierarchical system problems, and solve various problems in the process of system integration.

\section{The Characteristics of MES in the Steel Industry}

\subsection{The specialties of steel industry}

Iron and steel enterprises are continuous process enterprises. The production of steel is multi-workshop joint production with many production facilities and a high degree of automation. It has a large number of automation equipment, data acquisition systems and inspection systems. Among them, continuous production mainly includes blast furnace, sintering, coking and other workshops, and the discrete production mainly includes steelmaking, continuous casting, hot rolling and other workshops. In the steel industry, there are some problems urgently needs to be solved in MES: complex business management processes, uneven levels of automation at the physical level, the limitations of scalability and reconfiguration of system functions [7].

\subsection{MES integration characteristics of steel industry}

In view of the problems, take MES development of a large seamless steel tube plant in northwest China (hereinafter referred to as the seamless factory) as an example, we put forward two-layer structure model according to the independence of the MES integration and compatibility (Fig. 1).

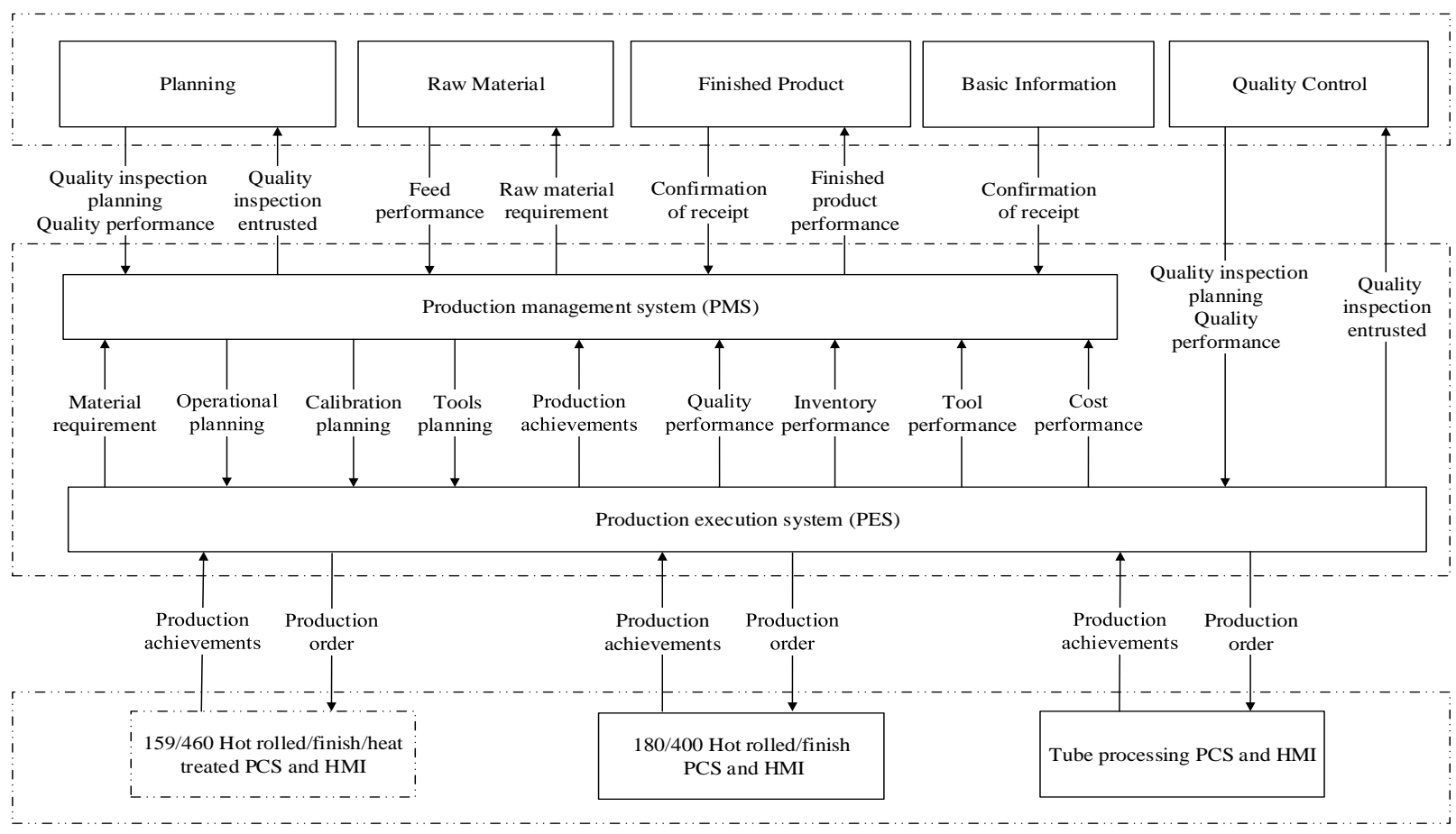

Fig. 1: System integration framework.

ERP includes functional modules such as production planning and management, inventory management, sales and distribution, financial management, quality management, etc. MES includes operation planning, production scheduling, material tracking, equipment management, quality management, process management, inventory management and other functions [8]. In the case of MES design, the function includes the plant 
management system (PMS) for plant-level cost center and production line-oriented factory execution system. It covers a wide range of functional modules, including production planning and management, and connection with the PCSs. In the design of MES, plant management system (PMS) is unified for management system in seamless steel tube plant, which is responsible for the centralized production management of whole plant. PMS is connected with the PES of each line, and the PES of each production line are connected with the corresponding PCS to realize the functions of production execution, material tracking and material consumption. The implementation of integrated PMS-PES forms the benign cycle of PDCA which is managed by seamless steel tube, so that the production management problems can be discovered and solved in time. Among them, PMS focuses on planning and implementation, and PES focuses on execution and inspection. With these functions, the system satisfies a lot of requirements, not only plant-level production line control and manufacturing management, but also corporate-level management. Although different steel companies have different functional requirements for MES, but on the whole, under the combination of steel-making order-oriented and inventory-oriented production, if MES- ERP integration environment are implemented, they will undertake the following functions respectively: ERP is responsible for basic information maintenance, production planning, quality management, inventory management, financial management and sales distribution. MES is responsible for production scheduling, manufacturing execution, material tracking and part of inventory management.

\section{MES-ERP Integration Design}

\subsection{MES-ERP design objectives}

There are several objectives of the design and implementation of MES-ERP: (1) Improve the efficiency of the company's overall utilization of information resources. (2) Arrange the system function module reasonably, coordinate the docking process, and realize the seamless connection. (3) Maximize data synchronization, realize real-time sharing of information, and provide timely and accurate data for production departments and management departments. (4) Establish a reasonable communication mechanism, and form a complete information chain between MES and ERP. Then a complete closed-loop system environment of planning, control, feedback and adjustment can be realized.

\subsection{System integration architecture}

In order to achieve information interaction between different levels of systems, we must balance the division of functions between MES and ERP, and establish effective ways of information interaction. The functions of production planning, basic information standardization, finished product management and raw material management of MES are replaced by ERP in the connection. ERP will take product inspection as a module, in this way, the basic information standardization, operation planning, quality control and other issues are sent to MES, which will be further processed by MES. To realize production control and supervision from the whole, raw material inventory is monitored in real-time. The main requirements of MES-ERP are shown in Table 1 (the upload message represents the information MES transmitted to ERP, and the download message represents the information ERP transmitted to MES).

Table 1: Interface requirements

\begin{tabular}{ccc}
\hline Interface & Module & Download Message/Upload Message \\
\hline MES.ERP1 & basic information & basic information maintenance information /without \\
MES.ERP2 & planning & plan, technology; order closing information /operation scheduling \\
& & plan receiving information \\
MES.ERP3 & raw material & raw material transfer information; raw material quality judgment \\
& & information /raw material inventory information; transfer library \\
MES.ERP4 & quality inspection & quality inspection plan information /sampling /supplementary \\
& & single dynamic communication \\
MES.ERP5 & finished product & transfer instructions /finished product delivery information \\
\hline
\end{tabular}




\subsection{Interface module design}

In order to meet the requirements of MES-ERP, the interface program is divided into five modules according to the function characteristics of MES. Each module is different from the others in communication mechanism. These five modules can be summarized as basic information module, planning module, raw material module, quality inspection module and finished product module.

- Basic information module

In order to realize the company's unified basic information standard, ERP will realize the unified management of five kinds of information includes PMS layer steel, steel grade, executive standard, steelmaking ingredient and internal control composition by interface MES.ERP1. This module interface is completely independent of the operation of MES, hence deploying services in the server background can save time costs. All of the five sub-modules of MES.ERP1 have added, modified and removed functions. The interface reads the intermediate database every $3 \mathrm{~min}$ and maintains the information directly to the MES database.

- Planning module

MES.ERP2 is the production planning interface, transmitting production plans to MES in the form of message, and directly updating to MES database can effectively save time and labor cost. After MES received new production plan, the plan will be further processed and sent to PCSs. The whole process can be divided into two processes: upload and download. The download messages mainly include production line operation plans, processes and orders, and order closing information. The upload messages include operation scheduling plans' receiving/canceling information, which are used to respond to the ERP project reception. MES.ERP2 has two functions of return and confirm. ERP can change production plan if it is not received If it has been received, the operation plan will be locked. If the operation plan is rejected or returned, feedbacks will be given to ERP to make changes to the plan and reissue it.

- Raw material module

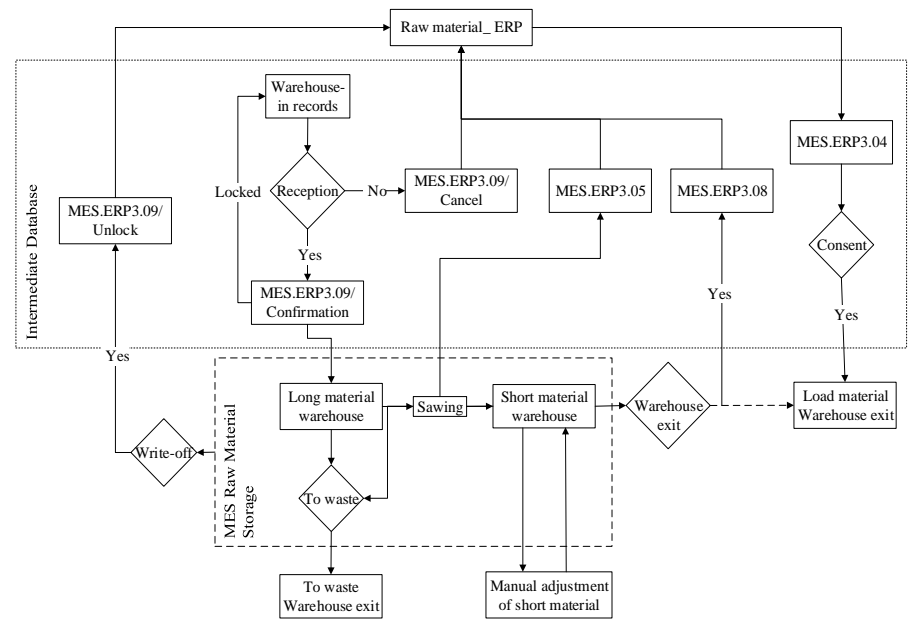

Fig. 2: Raw material interface functions.

Seamless factory, for example, each production line has its own raw material storage, so in the design process of raw material interface of seamless factory, the information of MES raw materials will be issued by ERP inventory when warehousing. In addition, the inventory dynamics of the raw materials are also strictly monitored by ERP. There are many operations in the raw material warehouse of MES, so the related data volume of MES.ERP3 is the largest and most complex. The data transmission of the corresponding raw material interface is shown in figure 2, among which the business process of tube inventory is most complicated. A total of nine kinds of events can trigger the MES to transfer data to ERP, when raw materials enter the warehouse, raw materials sawing, billets enter the heating furnace, return of raw materials after sawing, raw materials scraped after sawing, billets scraped after entering furnace, raw materials sentenced to waste, modify of steel grade and manual adjustment of warehouse. The above business processes [9 10] have a high requirement for the real-time and accuracy of data. 
- Quality inspection module

The quality management of steel should be unified and responsible by the quality inspection center of the company level. After samples and orders are submitted to ERP, inspectors can complete the inspection items in ERP, and return the results to MES through MES-ERP. MES reads once per 3min and updates them to the corresponding business database. The quality inspection plan is issued together with the operation plan.

- Finished product module

ERP is responsible for the management of all finished products of the enterprise. The business of the finished module MES.ERP5 can be seen as upload or download inventory changes messages. The finished module can be divided into two categories from the perspective of product purpose, one is the raw material used for other production lines and the other is the finished product. Among them, the finished product serves as the raw material for other production lines similar to the raw material interface, mainly to master the balance of materials. Businesses that can trigger these operations include warehousing, depot, transfer, and inventory adjustments.

\subsection{Implementation method}

In the process of the integration of ERP and MES in seamless factory, the implementation methods are divided into two parts, namely, the construction of database environment and the design of communication mechanism. These two parts complement each other to build the communication environment required for the MES-ERP interface.

Since ERP is the information portal of enterprise and MES is the execution system for the workshop, in the seamless factory, ERP and MES should adopt a more mature database technology. Because the differences in geographic location of the two systems, the database building adopts data sharing between heterogeneous databases. Oracle heterogeneous database can communicate and exchange data through the connection mechanism of the database itself, the direct way is: through "DB_Link" mechanism to establish the information exchange link between ERP intermediate database and MES database, which is a new oneto-one equivalent synonym structure, so as to achieve the consistency of transactions, to avoid the inaccuracy of data generated by power outages, network outages, etc.

ERP and MES communicate in the way of data transmission message, and the recognition of a message is based on the field identity and state. Each form has two fields of transmission code (form_id) and state (status), the former is used to distinguish between business area of the field (such as POP interface belongs to the operation plan, POPA belongs to the table, POPA12 is hot-rolled the main operating plans), and the latter is to distinguish the read state from that table (such as the table is set to 1 before it is read, changed to 0 and locked after read). In the operation rules of the intermediate database in ERP-MES, ERP can freely change or delete the intermediate database's information once the message is unlocked, otherwise, the state must be modified by MES before it can be operated. When MES finds out that the information is wrong or doesn't meet the production needs, MES needs to inform ERP, and then the original intermediate table will be modified or deleted by ERP. There are two ways to download data to the MES database: one is automatically updating the MES database directly (e.g., operation plan), the other is to be able to update the database after MES consent (such as MES can refuse raw materials for storage). State-based reading mode and demandbased loading mode together constitute the MES-ERP communication mechanism.

\section{System Implementation Effect}

With the implementation of MES and MES-ERP in the 159/460mm production lines of seamless factory, the practical application of the MES-ERP interface proved the stability, accuracy, fault tolerance and real time of system. The accuracy of the data reached more than $99 \%$, and the manual intervention rate basically dropped below $1 \%$, which proved the punctuality and accuracy of the data. In addition to fully meeting the business requirements, the current communication mechanism also guarantees that the connection and data synchronization can be restored after system crash due to power failure, shutdown, network failure, etc.

In order to meet the strategic goals of information construction of the corporation, the seamless factory basically achieved the purpose of data integration, integrated control, and opened the information island between MES, ERP and PCS by interfaces. The successful implementation of corporate data integrity and 
data resource integration has laid a solid foundation for the smooth operation of daily business and the accurate release of management decisions.

\section{Conclusion}

The integration of MES and ERP system is an essential stage for the development of information technology of iron and steel enterprises, which can greatly improve the management automation level of steel mills. At present, MES and ERP basically do not involve each other's functions in system design. Therefore, it is an urgent topic to study the integration method of MES-ERP and realize the complete integration of both systems.

This paper discusses and practices the mode and method of MES-ERP system integration in iron and steel enterprises. We mainly describe the core modules such as production management and inventory management. In the future, further study of the modules of equipment management, personnel management and cost management can be carried out.

\section{Acknowledgements}

This research is supported by National Natural Science Foundation of China (71701016, 71273025), Beijing Natural Science Foundation (9174038) and Humanity and Social Science Youth foundation of Ministry of Education of China (17YJC630143).

\section{References}

[1] Neves J M S D, Akabane G K, Marins F A S, et al. Deployment the MES (Manufacturing Execution System) aiming to improve competitive priorities of manufacturing[J]. Independent Journal of Management \& Production, 2015, 6(2): págs. 449-463.

[2] T.K. Li. A Review of The Manufacturing Execution System Models[J]. Metallurgical Industry Automation, 2003, 27(4):13-17.

[3] Nan Y E, Li-Feng X I. Real-time Improvement and System Integration of Manufacturing Execution System(MES)[J]. Industrial Engineering \& Management, 2005.

[4] Liu L, Yan G R, Lei Y. Study on Distributed Architecture, Information Integration and Access Control of Manufacturing Execution System[J]. Advanced Materials Research, 2013, 734-737:3207-3213.

[5] C.T. Shi, Z.X. Wu, L.X. Liu. MES Integration Solution of Seamless Steel Tube Plant of Baotou Steel[J]. Metallurgical Industry Automation, 2015(4):38-44.

[6] Govindaraju R, Putra K. A methodology for Manufacturing Execution Systems (MES) implementation[C]// IOP Conference Series: Materials Science and Engineering. IOP Publishing, 2016:012094.

[7] Z.L. Cheng, Y.Q. Fan. Design of Component-based Flexible MES in Metallurgical Industry[J]. Computer Integrated Manufacturing Systems, 2007, 13(3):490-496.

[8] Choi B K, Kim B H. MES (manufacturing execution system) architecture for FMS compatible to ERP (enterprise planning system)[J]. International Journal of Computer Integrated Manufacturing, 2002, 15(3):274-284.

[9] Z.X. Wu, T.K. Li, B.L. Wang. Order scheduling method of hot rolling seamless steel pipe with process route[J]. Computer Integrated Manufacturing Systems, 2016, 22(10):2355-2362.

[10] Z.X. Wu, T.K. Li, W.X. Zhang, B.L. Wang. Methods of hot-rolled batch planning for seamless steel tube with machine maintenance[J]. Control Theory and Applications, 2017, 34(9): 1250-1259. 